\section{Transmission electron microscopy for nanomedicine: novel applications for long-established techniques}

\author{
M. Malatesta \\ Department of Neuroscience, \\ Biomedicine and Movement, Section \\ of Anatomy and Histology, \\ University of Verona, Italy
}

\begin{abstract}
During the last twenty years, the research in nanoscience and nanotechnology has dramatically increased and, in the last decade, the interest has progressively been oriented towards biomedical applications, giving rise to a new field termed nanomedicine. Transmission electron microscopy is a valuable technique not only for the thorough physico-chemical characterization of newly synthesized nanoparticulates, but especially to explore the effects of nanocomposites on biological systems, providing essential information for the development of efficient therapeutic and diagnostic strategies. Thus, for the progress of nanotechnology in the biomedical field, experts in cell biology, histochemistry and ultramicroscopy should always support the chemists, physicists and pharmacologists engaged in the synthesis and characterization of innovative nanoconstructs.
\end{abstract}

\section{Introduction}

During the last twenty years, the research in nanoscience and nanotechnology has dramatically increased with more than 115,000 articles published in qualified journals since 1995; in the last decade, the interest has progressively been oriented towards biomedical applications (Figure 1), giving rise to a new field termed nanomedicine.

A plethora of nanoparticulates have been experimented for different applications: as drug delivery systems, contrast agents for in vivo imaging, sensors to specifically detect cells or organic components in the living body, sorting systems, scaffold components for tissue engineering, and many other innovative uses. ${ }^{1-5}$ The great versatility of nanomaterials offers, in fact, special advantages in biomedicine: their size (1 to $100 \mathrm{~nm}$ ) approaches the biomolecular scale, facilitating their interactions with the biological environment; in addition, they can be made of organic, inorganic or both materials, and assume various configurations (spheres, shells, rods, dishes, prisms, cubes, etc.), making them suitable tools for multiple purposes. Moreover, their surface can be functionalized allowing the targeting to specific cell types. ${ }^{6,7}$

Transmission electron microscopy (TEM) is a valuable technique for the thorough physicochemical characterization of newly synthesized nanoparticulates; actually, during the last ten years, more than 15,000 articles on nanoparticles include TEM among the used methods (https://www.ncbi.nlm.nih.gov/ pubmed/). TEM is also recently becoming a technique of choice to explore the effects of nanocomposites on biological systems: on a total of about 6,500 scientific articles on this subject in the last ten years, 2,400 (37\%) were published from 2014 to 2016 .

Generally, nanovectors are first tested using in vitro cultured cells, which ensures simple and controlled conditions as well as short experimental times; this also implies significantly reduced costs for the preliminary characterization of nanovectors, and a reduction in the number of laboratory animals for the following in vivo studies aimed at verifying their systemic suitability.

The first step of safety assessment of nanomaterials for biomedical use must encompass not only cell death evaluation but also any cell stress or damage at short, medium and long term, since cell necrosis or apoptosis may trigger an inflammatory response in the organism of the patient receiving the nanoparticulate system. ${ }^{8,9}$ Moreover, the localization and dynamic tracking of nanoparticulates inside the cells and tissues represent an essential information to design efficient administration strategies. Ultrastructural studies can proficiently be used to this aim.

\section{Ultrastructural morphology for nanotechnology}

To play their therapeutic or diagnostic function, nanoconstructs must interact with the biological environment, in primis with the cell. It is mandatory to know their behavior, from their ability to cross the plasma membrane, to their intracellular traffic up to their degradation route.

Thanks to its high resolution, TEM can provide unequivocal information on the uptake mechanism(s): nanocarriers have been observed making contact with the plasma membrane -either singly or as small groupsand entering the cell by endocytic processes; in the presence of large clusters of nanoparticulates, some cells are able to protrude pseudopods and internalize the clusters by
Correspondence: Manuela Malatesta,

Department of Neuroscience, Biomedicine and

Movement, Anatomy and Histology Section,

University of Verona, Strada Le Grazie 8, 37134

Verona, Italy. Tel. +39.045.8027569.

E-mail: manuela.malatesta@univr.it

Key words: Biodistribution; nanoparticles; nanotechnology; ultrastructure.

Received for publication: 19 November 2016. Accepted for publication: 27 November 2016 .

This work is licensed under a Creative Commons Attribution-NonCommercial 4.0 International License (CC BY-NC 4.0).

CCopyright M. Malatesta, 2016

Licensee PAGEPress, Italy

European Journal of Histochemistry 2016; 60:2751 doi:10.4081/ejh.2016.2751

phagocytosis; other nanovectors, especially of lipid nature, have been found to pass the biological membranes by fusion, thus avoiding the endosomal route. ${ }^{10-21}$ These different uptake mechanisms entail important functional consequences. In fact, endocytosis-mediated internalization, especially when receptors are involved, may reflect a limited uptake capability and a consequently low/slow intracellular accumulation of nanovectors. This is particularly true when nanocarriers are internalized as single units. On the other hand, phagocytosis (which often occurs together with endocytosis) allows high amounts of nanoparticulates to enter the cell, giving often rise to large cytoplasmic inclusions. Finally, membrane crossing by lipid raft-mediated endocytosis leads to a rapid and massive entrance of nanoparticulates, as a consequence of hydrophobic internalization through lipid membrane fusion or by uptake of lipophilic and anionic groups via scavenger receptor-mediated membrane fusion. ${ }^{22}$

These features must be taken into consideration when planning to use nanovectors, for example, as drug carriers or contrast agents or signaling systems. In particular, lipid rafts are typical of many human tumor cells, ${ }^{23,24}$ thus representing a potential advantage for the therapeutic/diagnostic utilization of lipid nanovectors.

It is worth noting that some nanoparticles may adhere each other through electrostatic or other binding interactions, and this may obviously affect their capability to be internalized by cells:12,16 TEM can provide information on these interactions, provided that the appropriate procedures for sample fixation and embedding are used to preserve the nanoparticles or nanoparticle clusters at the cell surface as well as in the extracellular space. 
The uptake mechanism strongly affects the intracellular fate of the nanoparticulates. In fact, the entrapment into endosomes (as it occurs when internalization is endocytosis- or phagocytosis-mediated) implies that internalized nanovectors will follow the endolytic route, undergoing degradation by the lysosomal enzymes. ${ }^{25}$ Some nanomaterials (especially cationic polymers) are able to escape endosomes due to a "proton sponge effect" which leads to the rupture of the nanoparticulateentrapping vacuole; ${ }^{26}$ this event does not generally induce cell damage probably because of the inability of lysosomal enzymes to be active at the cytosolic neutral $\mathrm{pH}$, or due to the rapid repair of the vesicle membrane preventing the efflux of lysosomal enzymes after the nanoparticles had been released.

Through this phenomenon, nanoparticulates may occur free in the cytosol and escape lysosomal degradation. When present free in the cytosol, nanoparticles can interact with cytoplasmic organelles possibly interfering with their functions. Free cytosolic nanoparticles have been demonstrated to enter the nucleus, ${ }^{27-32}$ either by passing through the nuclear pores $^{33}$ or being entrapped in the nuclear envelope when it reassembles at the end of mitosis; ${ }^{34}$ anyway, this event may have unpredictable consequences on the nucleusdriven cell activity. Generally, after a more or less prolonged period, cytosolic nanovectors are enclosed in autophagosomes; thus they reenter the lytic pathway and either undergo degradation or give rise to residual bodies, whose intracellular persistence depends on nanoparticulate amount and biodegradability. ${ }^{12,13,30,35,36}$ TEM provides detailed information on these events allowing to identify the different steps of the intracellular pathways and, importantly, to distinguish the presence of intact nanovectors or their remnants after enzymatic lysis: this latter finding is important to accurately describe the intracellular permanence of nanoparticles which cannot be properly obtained by light (namely confocal fluorescence) microscopy.

Nanovectors immediately occur free in the cytosol when they enter the cells by route other than the endosomal one, and they may persist therein for variable periods before undergoing autophagy and enzymatic degradation. Interestingly, ultrastructural observations recently allowed the visualization of the rapid cytosolic disassembly of liposomes shortly after their uptake: ${ }^{12}$ liposome components accumulate inside the lipid droplets (probably due to chemical affinity) and are finally extruded from the cells as lipid remnants.

In the case of drug-delivering nanocarriers, data on their intracellular persistence and degradation are useful to predict the fate of the loaded molecules and, therefore, to plan treat- ment strategies ensuring appropriate availability and sustainability of the therapeutic/diagnostic agents. Moreover, the intracellular persistence of free nanoparticulates or their remnants must be carefully considered for the evaluation of cell stress, especially when multiple administrations are planned.

When nanoconstructs are tested ex vivo or in vivo, the fine analysis of their distribution in the different tissues becomes crucial to plan their biomedical application: the identification of the tissue components where the nanocomposites localize (i.e., specific cell types, the amorphous or fibrillar components of the extracellular matrix, the blood vessels, etc.) is important to evaluate the capability of the nanovectors to reach the expected biological target and, consequently, to predict its potential therapeutic/diagnostic role. In particular, the ability to cross the biological barriers is crucial to select the most suitable administration routes and protocols (concentrations, doses, etc.). It is in fact known that a significant number of newly synthesized nanocomposites successfully tested in in vitro models proved to be unsuitable when applied in vivo because of the systemic defense. ${ }^{37}$

In addition to nanovectors tracking, TEM play a key role in the identification of cell structural and molecular alterations induced by the nanoparticulate: membrane breakage, mitochondria swelling or shrinkage, changes in the length or shape of the mitochondrial cristae, reorganization of the endoplasmic reticulum and Golgi apparatus, rearrangement of the nuclear domains are all undetectable features at the lower resolution of light microscopy.

\section{Beyond ultrastructural morphology}

The visualization of nanomaterials at TEM depends on their chemical composition. Nanoparticulates containing heavy atoms are more electron dense than those containing atoms of lower mass. Consequently, inorganic nanoconstructs (e.g., gold, silver, iron oxide nanoparticles, silica-based nanoparticles) are easily detectable inside cells and tissue due to their intrinsically high electron density and do not need any particular staining procedure to enhance their contrast. On the other hand, nanoparticles made of organic material (e.g. lipid, chitosan, poly(lactic-co-glycolic acid), albumin) are characterized by a moderate electron density that makes them hardly distinguishable in the cytosol or inside the lysosomal compartments. Lipid-based nanoconstructs can be easily made more electron dense by osmium tetroxide post-fixation. To unequivocally detect polymeric and proteinaceous nanoparticles in the intracellular milieu, the problem can be overcome by applying histochemical procedures for the photo-oxidation of diaminobenzidine (DAB): this technique allows to convert the fluorescent signal of fluorochromes into a stable electron dense, finely granular reaction product visible at the high spatial resolution of TEM. ${ }^{38}$ This method proved to be so efficient to enable detecting fluorescently labelled nanoparticles even inside residual bodies (i.e., after the lytic action of lysosomal enzymes had transformed the nanoparticles into morphologically unrecognizable remnants). ${ }^{11,13,39}$ DAB photo-0xidation has been applied so far to nanocomposites

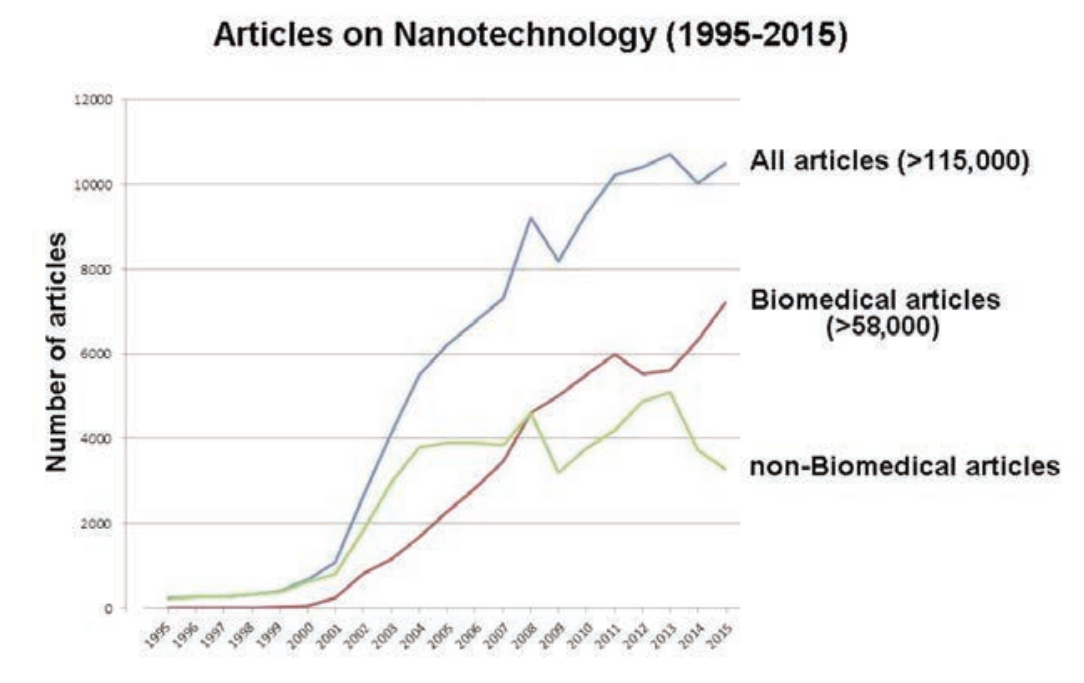

Figure 1. Number of articles on nanotechnology during the last twenty years (from the Web of Science database). 
synthesized with fluorescent components (e.g., fluorescein isothiocyanate, Nile red or rhodamine), but its application may be foreseen also for autofluorescing nanocomposites. ${ }^{40-44}$

Nanoparticles have been unambiguously identified at the ultrastructural level also by means of correlative microscopy approaches: advanced TEM methods (conventional, immuno and energy-filtered electron microscopy, and electron tomography) were used in combination with light microscopy to analyze the distribution of different nanoparticles in tissues and cells, ${ }^{45}$ fluorescence and electron microscopy (TEM and scanning transmission electron microscopy, STEM) allowed the ultrastructural identification of quantum dots inside fibroblasts ${ }^{46}$ and human pathological tissues, ${ }^{47}$ interferometric photo-activated localization microscopy and electron microscopy were correlated to describe the intracellular distribution of gold nanoparticles ${ }^{48}$ correlative optical microscopy and scanning electron microscopy (SEM) were used to locate gold-core nanoparticles in brain tumors, ${ }^{49}$ cryo-soft X-ray tomography provided information on three-dimentional interactions of super-paramagnetic iron oxide nanoparticles with breast cancer cells; ${ }^{50}$ uptake and intracellular distribution within breast cancer cells of $\mathrm{ZnO} 0$-based nanoparticles were analysed combining dynamic confocal imaging, low resolution bright field TEM and dark field STEM; 51 TEM and Serial Block Face SEM (SBF SEM) were used to quantify the intracellular uptake of quantum dots. ${ }^{18}$

Nanoparticles have been demonstrated to be easily detectable after DAB photo-oxidation not only in biological samples processed for conventional fine morphology (aldehyde fixation, osmium tetroxide post-fixation and epoxy resin embedding), but also for ultrastructural cytochemistry and immunocytochemistry (aldehyde fixation and acrylic resin embedding), and DAB photo-oxidation was demonstrated to be successfully combined with immunoelectron microscopy. ${ }^{52}$

Therefore, the contribution of TEM to the development of biomedical nanotechnology is not limited to the conventional morphological approach aimed at precisely localizing the nanoparticulates, but encompasses molecular analyses in situ, which enables evaluation of functional effects of nanoconstructs administration.

\section{Concluding remarks}

The widespread application of ultrastructural analyses in nanotechnology clearly demonstrates the great (still only partially exploited) potential of electron microscopy in this field.
Looking at the scientific literature, most of the newly synthesized nanoconstructs for biomedical use have been aimed at treating cancer (about 22,000 peer reviewed scientific articles in the last ten years; https://www.ncbi. nlm.nih.gov/pubmed/); in these investigations, light (brightfield and fluorescence) microscopy was often exclusively used to assess the efficacy of nanoparticle administration, based on the occurrence of cell death or tissue necrosis. The ultrastructural reports in the literature are less numerous, and most of them are strictly morphological and focused on the spatial relationships of nanoconstructs with the plasma membrane or the cytoplasmic organelles, as the causal events for cell damage and death.

It is worth noting that the informational capacity of TEM is much greater, especially when cytochemical techniques are used to elucidate dynamic cellular processes ${ }^{53-55}$ or to spatially localize single molecules or molecular complexes. ${ }^{56,57}$ This approach would especially be convenient for studying the effects of nanovector-based therapies aimed at curing diseased cells which are to be preserved and functionally repaired (e.g. highly differentiated non-cycling cells such as myofibres and neurons, in muscular or neurodegenerative diseases); indeed, ultrastructural cytochemistry enables to finely describe the cellular morphofunctional changes induced by drug-delivering nanovectors, providing evidence for the restored healthy features and for the possible occurrence of undesired negative side-effects. To properly describe the cellular post-treatment events, it is crucial the selection of the most suitable cell model systems, the appropriate procedures for the preparation of microscopical samples, and the specific histochemical analyses to be used. Thus, for the progress of nanotechnology in the biomedical field, experts in cell biology, histochemistry and ultramicroscopy should always support the chemists, physicists and pharmacologists engaged in the synthesis and characterization of innovative nanoconstructs.

\section{References}

1. Lim CT, Han J, Guck J, Espinosa H. Micro and nanotechnology for biological and biomedical applications. Med Biol Eng Comput 2010;48:941-3.

2. Bobo D, Robinson KJ, Islam J, Thurecht KJ, Corrie SR. Nanoparticle-based medicines: a review of FDA-approved materials and clinical trials to date. Pharm Res 2016;33:2373-87.

3. Fernandes RS, Dos Santos Ferreira D, de Aguiar Ferreira C, Giammarile F, Rubello
D, de Barros AL. Development of imaging probes for bone cancer in animal models. A systematic review. Biomed Pharmacother 2016;83:1253-64.

4. Mehta A, Ghaghada K, Mukundan S Jr. Molecular imaging of brain tumors using liposomal contrast agents and nanoparticles. Magn Reson Imaging Clin N Am 2016;24:751-63.

5. Soica C, Coricovac D, Dehelean C, Pinzaru I, Mioc M, Danciu C, et al. Nanocarriers as tools in delivering active compounds for immune system related pathologies. Recent Pat Nanotechnol. 2016;10:128-45.

6. Friedman AD, Claypool SE, Liu R. The smart targeting of nanoparticles. Curr Pharm 2013;19:6315-29.

7. Dehaini D, Fang RH, Zhang L. Biomimetic strategies for targeted nanoparticle delivery. Bioeng Transl Med 2016;1:30-46.

8. Rock KL, Kono H. The inflammatory response to cell death. Annu Rev Pathol 2008;3:99-126.

9. Kono H, Kimura Y, Latz E. Inflammasome activation in response to dead cells and their metabolites. Curr Opin Immunol 2014;30:91-8.

10. Zhang LW, Bäumer W, Monteiro-Riviere NA. Cellular uptake mechanisms and toxicity of quantum dots in dendritic cells. Nanomedicine (Lond) 2011;6:777-91.

11. Malatesta M, Giagnacovo M, Costanzo M, Conti B, Genta I, Dorati R, et al. Diaminobenzidine photoconversion is a suitable tool for tracking the intracellular location of fluorescently labelled nanoparticles at transmission electron microscopy. Eur J Histochem 2012;56:e20.

12. Costanzo M, Carton F, Marengo A, Berlier G, Stella B, Arpicco S, et al. Fluorescence and electron microscopy to visualize the intracellular fate of nanoparticles for drug delivery. Eur J Histochem 2016;60:2640.

13. Costanzo M, Scolaro L, Berlier G, Marengo A, Grecchi S, Zancanaro C, et al. Cell uptake and intracellular fate of phospholipidic manganese-based nanoparticles. Int J Pharm 2016;508:83-91.

14. Boyles MS, Kristl T, Andosch A, Zimmermann M, Tran N, Casals E, et al. Chitosan functionalisation of gold nanoparticles encourages particle uptake and induces cytotoxicity and pro-inflammatory conditions in phagocytic cells, as well as enhancing particle interactions with serum components. J Nanobiotechnology 2015;13: 84.

15. Butler KS, Peeler DJ, Casey BJ, Dair BJ, Elespuru RK. Silver nanoparticles: correlating nanoparticle size and cellular uptake with genotoxicity. Mutagenesis 2015;30:577-91.

16. Poussard S, Decossas M, Le Bihan 0, 
Mornet S, Naudin G, Lambert 0 . Internalization and fate of silica nanoparticles in $\mathrm{C} 2 \mathrm{C} 12$ skeletal muscle cells: evidence of a beneficial effect on myoblast fusion. Int J Nanomedicine 2015;10:147992.

17. Alfranca G, Artiga A, Stepien G, Moros M, Mitchell SG, de la Fuente JM. Gold nanoprism-nanorod face off: comparing the heating efficiency, cellular internalization and thermoablation capacity. Nanomedicine (Lond) 2016;11:2903-16.

18. Hondow N, Brown MR, Starborg T, Monteith AG, Brydson R, Summers HD, et al. Quantifying the cellular uptake of semiconductor quantum dot nanoparticles by analytical electron microscopy. J Microsc 2016;261:167-76.

19. Lopes VR, Loitto V, Audinot JN, Bayat N, Gutleb AC, Cristobal S. Dose-dependent autophagic effect of titanium dioxide nanoparticles in human HaCaT cells at non-cytotoxic levels. J Nanobiotechnology 2016;14:22.

20. Messerschmidt C, Hofmann D, Kroeger A, Landfester K, Mailänder V, Lieberwirth I. On the pathway of cellular uptake: new insight into the interaction between the cell membrane and very small nanoparticles. Beilstein J Nanotechnol 2016;7:1296-311.

21. Zielinska E, Tukaj C, Radomski MW, Inkielewicz-Stepniak I. Molecular mechanism of silver nanoparticles-induced human osteoblast cell death: protective effect of inducible nitric oxide synthase inhibitor. PLoS One 2016;11:e0164137.

22. Lanza R, Langer R, Vacanti JP. Principles of tissue engineering. 4th ed. New York: Academic Press; 2011.

23. Mollinedo F, Gajate C. Lipid rafts as major platforms for signaling regulation in cancer. Adv Biol Regul 2015;57:130-46.

24. Nicolson GL. Cell membrane fluid-mosaic structure and cancer metastasis. Cancer Res 2015;75:1169-76.

25. Panyam J, Zhou WZ, Prabha S, Sahoo SK, Labhasetwar V. Rapid endolysosomal escape of poly(DL-lactide-coglycolide) nanoparticles: implications for drug and gene delivery. FASEB J 2002;16:1217-26.

26. Varkouhi AK, Scholte M, Storm G, Haisma HJ. Endosomal escape pathways for delivery of biologicals. J Control Release 2011;151: 220-8.

27. Nabiev I, Mitchell S, Davies A, Williams Y, Kelleher D, Moore R, et al. Nonfunctionalized nanocrystals can exploit a cell's active transport machinery delivering them to specific nuclear and cytoplasmic compartments. Nano Lett 2007;7:3452-61.

28. Colonna C, Dorati R, Conti B, Modena T, Biggiogera M, Spedito A, et al. Induction of an in vitro reversible hypometabolism through chitosan-based nanoparticles. J Microencapsul 2011;28:229-39.

29. Malatesta M, Galimberti V, Cisterna B, Costanzo M, Biggiogera M, Zancanaro C. Chitosan nanoparticles are efficient carriers for delivering biodegradable drugs to neuronal cells. Histochem Cell Biol 2013;141: 551-8.

30. Malatesta M, Grecchi S, Chiesa E, Cisterna B, Costanzo M, Zancanaro C. Internalized chitosan nanoparticles persist for long time in cultured cells. Eur J Histochem 2015;59:2492.

31. Braakhuis HM, Gosens I, Krystek P, Boere JA, Cassee FR, Fokkens PH, et al. Particle size dependent deposition and pulmonary inflammation after short-term inhalation of silver nanoparticles. Part Fibre Toxicol 2014;11:49.

32. Zhang X, Shastry S, Bradforth SE, Nadeau JL. Nuclear uptake of ultrasmall gold-doxorubicin conjugates imaged by fluorescence lifetime imaging microscopy (FLIM) and electron microscopy. Nanoscale 2015;7:240-51.

33. Hinde E, Thammasiraphop K, Duong HT, Yeow J, Karagoz B, Boyer C, et al. Pair correlation microscopy reveals the role of nanoparticle shape in intracellular transport and site of drug release. Nat Nanotechnol 2016. doi: 10.1038/nnano.2016.160.

34. Guan M, Zhu Q, Liu Y, Bei Y-Y, Gu Z-L, Zhang X-N, et al. Uptake and transport of a novel anticancer drug-delivery system: lactosyl-norcantharidin-associated $\mathrm{N}$ trimethyl chitosan nanoparticles across intestinal Caco-2 cell monolayers. Int J Nanomedicine 2012;7:1921-30.

35. Barthel AK, Dass M, Dröge M, Cramer JM, Baumann D, Urban M, et al. Imaging the intracellular degradation of biodegradable polymer nanoparticles. Beilstein J Nanotechnol 2014;5:1905-17.

36. Mazuel F, Espinosa A, Luciani N, Reffay M, Le Borgne R, Motte L, et al. Massive intracellular biodegradation of iron oxide nanoparticles evidenced magnetically at single-endosome and tissue levels. ACS Nano 2016;10:7627-38.

37. Patil US, Adireddy S, Jaiswal A, Mandava S, Lee BR, Chrisey DB. In vitro/in vivo toxicity evaluation and quantification of iron oxide nanoparticles. Int J Mol Sci 2015; 16:24417-50.

38. Maranto AR. Neuronal mapping: a photooxidation reaction makes Lucifer yellow useful for electron microscopy. Science 1982;217:953-5.

39. Malatesta M, Pellicciari C, Cisterna B, Costanzo M, Galimberti V, Biggiogera M, et al. Tracing nanoparticles and photosensitizing molecules at transmission electron microscopy by diaminobenzidine photooxidation. Micron 2014;59C:44-51.

40. Croce AC, Bottiroli G. Autofluorescence spectroscopy and imaging: a tool for biomedical research and diagnosis. Eur $\mathrm{J}$ Histochem 2014;58:2461.

41. Qiao H, Sun M, Su Z, Xie Y, Chen M, Zong L, et al. Kidney-specific drug delivery system for renal fibrosis based on coordination-driven assembly of catechol-derived chitosan. Biomaterials 2014;35:7157-71.

42. Shen Z, Ma X, Wu A. Gd203 nanocrystalbased autofluorescent composite nanoparticles as T1-weighted contrast agents. J Control Release 2015;213:e147-8.

43. Wei W, Lv P, Ma G. Oral delivery of protein and anticancer drugs by uniform-sized chitosan micro/nanoparticles with autofluorescent property. J Control Release 2015;213: el11.

44. Cai B, Rao L, Ji X, Bu LL, He Z, Wan D, et al. Autofluorescent gelatin nanoparticles as imaging probes to monitor matrix metalloproteinase metabolism of cancer cells. J Biomed Mater Res A 2016;104:2854-60.

45. Mühlfeld C, Rothen-Rutishauser B, Vanhecke D, Blank F, Gehr P, Ochs M. Visualization and quantitative analysis of nanoparticles in the respiratory tract by transmission electron microscopy. Part Fibre Toxicol 2007;4:11.

46. Dukes MJ, Peckys DB, de Jonge N. Correlative fluorescence microscopy and scanning transmission electron microscopy of quantum-dot-labeled proteins in whole cells in liquid. ACS Nano 2010;4:4110-6.

47. Killingsworth MC, Bobryshev YV. Correlative light- and electron microscopy using quantum dot nanoparticles. J Vis Exp 2016;114. doi: 10.3791/54307.

48. Shtengel G, Wang Y, Zhang Z, Goh WI, Hess HF, Kanchanawong P. Imaging cellular ultrastructure by PALM, iPALM, and correlative iPALM-EM. Methods Cell Biol 2014;123:273-94

49. Kempen PJ, Kircher MF, de la Zerda A, Zavaleta CL, Jokerst JV, Mellinghoff IK, et al. A correlative optical microscopy and scanning electron microscopy approach to locating nanoparticles in brain tumors. Micron 2015;68:70-6.

50. Chiappi M, Conesa JJ, Pereiro E, Sorzano C0, Rodríguez MJ, Henzler K, et al. Cryosoft X-ray tomography as a quantitative three-dimensional tool to model nanoparticle:cell interaction. J Nanobiotechnology 2016;14:15.

51. Othman BA, Greenwood C, Abuelela AF, Bharath AA, Chen S, Theodorou I, et al. Correlative light-electron microscopy shows RGD-targeted Zn0 nanoparticles dissolve in the intracellular environment 
of triple negative breast cancer cells and cause apoptosis with intratumor heterogeneity. Adv Healthc Mater 2016;5:131025.

52. Malatesta M, Zancanaro C, Costanzo M, Cisterna B, Pellicciari C. Simultaneous ultrastructural analysis of fluorochromephotoconverted diaminobenzidine and gold immunolabelling in cultured cells. Eur J Histochem 2013;57:e26.

53. Polishchuk EV, Polishchuk RS. Analysis of
Golgi complex function using correlative light-electron microscopy. Methods Cell Biol 2013;118:243-58.

54. Fossati M, Borgese N, Colombo SF, Francolini M. Visualization of Endoplasmic Reticulum Subdomains in Cultured Cells. J Vis Exp 2014;84:e50985.

55. Grecchi S, Malatesta M. Visualizing endocytotic pathways at transmission electron microscopy via diaminobenzidine photooxidation by a fluorescent cell-membrane dye. Eur J Histochem 2014;48:2449.

56. Pellicciari C. Impact of histochemistry on biomedical research: looking through the articles published in a long-established histochemical journal. Eur J Histochem 2014;58:2474.

57. Pellicciari C. Histochemistry as an irreplaceable approach for investigating functional cytology and histology. Eur J Histochem 2013;57:e41. 